hypertension, with conflicting results. Kazakans of the pasturing area in Xinjiang, China, have higher prevalence of hypertension and mean blood pressure than other populations of Xinjiang. But up to now no study in this population. To investigate the relationship between the $G(+252)$ A polymarphism of ADRB2 gene and essential hypertension in Kazaks of Xinjiang.

Method A total of 276 patients with confirmed hypertensives and 157 healthy control were genotyped for the G (+252)A by PCR restriction fragment length polymorphism (PCR-RFLP) analysis.

Result Compared with control group, there was no significant difference in the distribution of genotypes and allele frequency of $G$ $(+252)$ A polymorphisms in $\mathrm{EH}$ group $(\mathrm{p}>0.05)$. In addition, age, body mass index, systolic blood pressure and diastolic blood pressure had no significant difference in the groups classified according to genotypes $(\mathrm{p}>0.05)$.

Conclusion G (+252)A polymorphism of $\beta 2$-AR gene was not related with essential hypertension in Kazaks of XinJiang.

\section{e0163 THE ASSOCIATION OF FURIN GENE CODING POLYMORPHISM WITH ESSENTIAL HYPERTENSION IN KAZAKAN OF XINJIANG}

doi:10.1136/hrt.2010.208967.163

Li Nanfang, Luo Wenli, Yao Xiaoguang, Yan Zhitao, Zhang Juhong. Department of Echocardiography, Center of the Medical Ultrasound, The First Affiliated Hospital of Xinjiang Medical University, Urmuqi, China

Introduction The proprotein processing enzyme furin is involved in many blood pressure-regulating factors. In the Golgi, furin appears to activate $\mathrm{ENaC}$. Thus the gene of furin (FUR) may be a candidate gene of hypertension. We investigate the relationship between Furin gene polymorphism and hypertension in Xinjiang Kazakans.

Methods 924 Kazakans was selected randomly from the pasturing area aged in Xinjiang (422 hypertensives, 502 normotensive controls). We sequenced the coding regions of FUR in 94 hypertensive individuals to identify genetic variations of FUR. Genotyping by the TaqMan-PCR method was performed for common SNPs. The possible relationship between the polymorphism and hypertension of Kazakan was analysed.

Results 1 . Direct sequencing from 94 hypertension subjects identified 6 SNPs in the Furin gene promoter. 2. The genotypes and allele frequencies of $-229 \mathrm{C} \rightarrow \mathrm{T}, 12690 \mathrm{G} \rightarrow \mathrm{C}$ polymorphisms are not significant between these two groups. 3. There was no significance of blood pressure among the three genotypes of $-229 \mathrm{C} \rightarrow \mathrm{T}$, $12690 \mathrm{G} \rightarrow \mathrm{C}$ polymorphism. In multiple logistic analyses, the genotypes of the $-229 \mathrm{C}>\mathrm{T}$ were excluded as independent variables. None of haplotypes composed of $-229 \mathrm{C} \rightarrow \mathrm{T}$ and $12690 \mathrm{G} \rightarrow \mathrm{C}$ was significantly different in $\mathrm{EH}$ and controls.

Conclusion The Furin gene $-229 \mathrm{C} \rightarrow \mathrm{T}, 12690 \mathrm{G} \rightarrow \mathrm{C}$ polymorphism might not be associated with essential hypertension in Kazakans.

\section{e0164 DYSREGULATION OF MIR-1 AFTER LEFT VENTRICULAR HYPERTROPHY REVEALS A ROLE OF MIR-1 IN VENTRICULAR ARRHYTHMIA OCCURRED IN THE HYPERTENSIVE HEART}

doi:10.1136/hrt.2010.208967.164

Ying Huang, Weifeng Wu. Department of Cardiology, The First Affiliated Hospital of Guangxi Medical University, Nanning, China

Clinical studies have shown that the rate of VA in hypertensive patients with LVH was several times higher than the normaltensive ones. Currently the mechanism of VA due to LVH is not yet fully clarified. Growing evidences indicate that microRNAs (miRNAs or miRs) are regulators of gene expression, which are becoming increasingly recognised as important regulators of heart function and diseases. Here we observed that miR-1 was striking because of its more than $(2.08 \pm 0.21)$ fold increased in the spontaneously hypertensive rat model (SHRs) with LVH. miR-1 overexpression slowed conduction and depolarised the cytoplasmic membrane by post-transcriptionally repressing Kir2.1 and connexin 43(Cx43), and this likely accounts at least in part for its arrhythmogenic potential. Then we confirmed that in vivo suppression of miR-1 in SHRs could upgrade $\mathrm{Cx} 43$ and Kir2.1 protein level. Our data show that miR-1 is a key regulator of cardiac hypertrophy formation and VA due to $\mathrm{LVH}$, suggesting its attractive therapeutic application in ventricular arrhythmia occurred in the hypertensive heart.

\section{e0165 GENETIC VARIATION OF NEDD4L IS ASSOCIATED WITH ESSENTIAL HYPERTENSION IN FEMALE KAZAKH GENERAL POPULATION}

doi:10.1136/hrt.2010.208967.165

Li Nanfang, Wang Hongmei, Hong Jing, Yao Xiaoguang. Hypertension Unit of the People's Hospital of Xinjiang Uygur Autonomous Region, The Institute of Hypertension of Xinjiang, The Center of Hypertension of the People's Hospital of Xinjiang Uygur Autonomous Region, Urumqi, China

Introduction NEDD4L is a candidate gene for hypertension on both functional and genetic grounds. The study is to investigate the relationships between the variation of NEDD4L and essential hypertension (EH) in Kazakh in China.

Methods We screened the promoter and exons of NEDD4L in 94 Kazakh hypertensive individuals to identify representative variations. Then the representative variations were genotyped in a Kazakh general population, a case-control study was conducted.

Results We did not identify any functional mutations in functional regions of NEDD4L. Three representative variations (296921296923delTTG, rs2288774, rs2288775) were successfully genotyped in Kazakh population. The distribution of the dominant model (AA vs $A G+G G)$ of rs2288775, the additive model and recessive model (II+ID vs DD) of 296921-296923delTTG differed significantly between case and control in female $(p=0.040, p=0.024$ and $p=0.007$ respectively). Logistic regression analysis showed that rs2288775 and 296921-296923delTTG were significantly associated with hypertension ( $\mathrm{rs} 2288775$ : OR $=1.479,95 \%$ CI 1.011 to 2.064 , $p=0.044 ; 296921-296923$ delTTG: $O R=1.908,95 \%$ CI 1.020 to 3.568 , $\mathrm{p}=0.043$ ) in female. The frequency of $\mathrm{D}-\mathrm{C}-\mathrm{G}$ haplotype was significantly higher for case than for control in female $(p=0.020)$.

Conclusions The genetic variations of NEDD4L may be associated with EH in female Kazakh.

\section{e0166 VARIATION OF ADRB2 IS ASSOCIATED WITH COMMON RISK FACTORS FOR CARDIOVASCULAR}

doi:10.1136/hrt.2010.208967.166

Wang Hongmei, Li Nanfang, Hu Junli, Liu Hui. Hypertension Unit of The People's Hospital of Xinjiang Uygur Autonomous Region, The Institute of Hypertension of Xinjiang, The Center of Hypertension of The People's Hospital of Xinjiang Uygur Autonomous Region, Urumqi, China

Introduction Hypertension, overweight/obesity and dyslipidemia are common risk factors for cardiovascular disease. $\beta 2$-adrenergic receptor (ADRB2) regulates blood pressure, lipid mobilisation, and energy expenditure.

Methods A cross-sectional study was conducted in Kazakh of Xinjiang (169 males, 238 females) aged 30 to 60 years. The widely studied polymorphisms A46G, C79G, C491T and A523C in ADRB2 gene were selected to be genotyped by PCR-RFLP. 